\title{
¿Existen realmente los ensambles y ensamblajes ecológicos?
}

\section{Julián Monge-Nájera}

Laboratorio de Ecología Urbana, Vicerrectoría de Investigación, Universidad Estatal a Distancia, 2050 San José, Costa Rica; julianmonge@gmail.com

\author{
Recibido 23-III-2015. Corregido 01-V-2015. Aceptado 06-V-2015.
}

\begin{abstract}
Do biological assemblages and ensembles really exist? In recent years the terms assemblage and ensemble have become more frequent in the ecological literature. After analyzing the definitions of the words in the French original, as well as their use in English and Spanish and opinions on how they can be defined, I conclude that the words assemblage and ensemble rarely improve scientific communication and can be eliminated from most ecological articles. The few justified exceptions are articles in which the emphasis is on how taxonomically close species interact trophically. Rev. Biol. Trop. 63 (3): 575-577. Epub 2015 September 01.
\end{abstract}

Key words: terminology, correct use of technical terms, definition of ensemble and assemblage in biology, ecological ensemble.

En años recientes ha aparecido la palabra "ensamble" (con menos frecuencia, "ensamblaje") en la literatura biológica en castellano. Algunos ejemplos:

- Patrones de distribución espacial de ensambles de macroinvertebrados bentónicos de un sistema fluvial.

- Implicaciones del ensamblaje de especies y grupos funcionales en dinámica de vegetación.

- Estructura de un ensamblaje íctico asociado a fondos duros.

- Distribución espacial del ensamble de peces intermareales de una zona costera.

- Ensamblajes de peces arrecifales y su relación con el hábitat.

- Estructura gremial y organización de un ensamble de aves.

- Tamaño corporal en vertebrados y patrones a nivel de ensamblaje.

- Ecología del ensamble de micromamíferos en un agroecosistema.
El diccionario de la Real Academia Española no define ensamblaje pero refiere a ensamblar, indicando que proviene del francés antiguo ensembler, "Unir, juntar, ajustar, especialmente piezas de madera". No presenta otras definiciones.

En español estas palabras son calcos crudos de la palabra francesa assemblage, usada en inglés en casos como los siguientes:

- Comparison of fish assemblages between two reefs.

- Bat assemblage in areas subjected to natural regeneration.

- Temporal turnover and the maintenance of diversity in ecological assemblages.

- Resemblance in phylogenetic diversity among ecological assemblages.

- Assessing the indicator properties of species assemblages.

- Functional organization of stream fish assemblages.

- The reality of ecological assemblages: A Palaeo-ecological puzzle. 
El Diccionario Larousse define assemblage como «Tout formé d'éléments assemblés: Un livre est un assemblage de cahiers. Réunion inattendue ou disparate de personnes, de choses; collection, mélange» (larousse.fr), definición idéntica a la que da el Oxford (oxforddictionaries.com); pero hay un caso biológico en el Free Dictionary, el cual la define como «varias cosas agrupadas o consideradas como un todo» e incluye el ejemplo «toda la vida animal en una región o periodo particular; la fauna de China» (thefreedictionary.com).

Cooke (1984) define el ensamblaje como un «conjunto de especies que habitan un área particular cuando las interacciones entre especies, si las hay, no se especifican», definición que es la base de las presentadas posteriormente, por ejemplo por la FAO (Fisheries Glossary, fao.org/fi/glossary/default.asp). Moreira, Huising and Bignell (2012, p. 110) dan una definición diferente: «Los ensambles de especies son grupos de especies relacionadas taxonómicamente, de un hábitat o área geográfica específica. Algunas veces se refieren a 'comunidades de especies' pero este término no es correcto, pues dichos ensambles son delimitados por una clasificación taxonómica basada en historia y filogenia y no en relaciones ecológicas...».

Así, para Moreira et al. (2012) los ensambles son grupos taxonómicos, no ecológicos; sin embargo este concepto no calza con el uso común en español, que es grupos de especies que viven en un mismo lugar (e.g. Quijada y Cáceres 2000), más acorde con la definición inglesa de Cooke (1984). En un libro que trata específicamente el tema, Analysis of Ecological Communities, McCune, Grace y Urban (2002) afirman: «La palabra 'ensamblaje' se ha usado a menudo en el sentido de comunidad concreta. Esta palabra no solo resulta innecesariamente retorcida para un concepto simple, sino que tiene connotaciones indebidas. Para algunos implica que las especies son independientes y no interactúan». Tal vez el caso más defendible de una especie que está en un sitio sin ser parte de las relaciones ecológicas sea el de un ave migratoria que sobrevuela un ecosistema, pero esa falta de interacción no se ha demostrado y, en general, dado que como regla las especies de un ecosistema sí interactúan directa o indirectamente (Verhoef y Morin, 2009), el concepto de ensamble ecológico parece complicar más que aportar a nuestra comprensión de la naturaleza.

Una aclaración particularmente útil es la de Fauth et al. (1996), quienes usaron un diagrama de Venn para distinguir entre las palabras inglesas community, guild, assemblage y ensemble. Definen assemblage como el grupo de especies taxonómicamente cercanas que dentro de una comunidad usan recursos diferentes (ensemble si se concentran en un mismo recurso).

Aunque Fauth et al. (1996) no abundan en ejemplos, podemos considerar uno aquí. Si tenemos por ejemplo medio centenar de especies de aves que, en un lugar de la selva tropical lluviosa se alimentan unas de semillas, otras de lagartijas y otras de néctar, tenemos un assemblage (que podríamos traducir como "asamblea"). Pero los colibríes de esa "asamblea", al pertenecer todos al gremio de los nectarívoros, forman un subconjunto que podemos llamar ensemble ("conjunto").

Ya hace varias décadas Fabian M. Jaksić advirtió que diversos términos ecológicos eran mal usados en la literatura científica; por ejemplo comunidad en gran número de artículos con títulos como "La comunidad de peces de ..." y "Comunidades de aves en ...". El examen de muchos de ellos revela que no calzan con el concepto de comunidad (especies de varios grupos taxonómicos que interactúan tróficamente; Jaksić 1981, Verhoef y Morin 2009). Lo mismo ocurre con assemblage y ensemble.

Debido a que las palabras assemblage y ensemble tienen traducciones adecuadas al español, como asamblea y conjunto, no recomiendo el uso de esos galicismos importados por vía del inglés. Además insto a usar esas versiones castellanas sólo en los contados casos en que sean realmente necesarias para transmitir, respectivamente, las ideas de especies emparentadas que interactúan sobre varios recursos (asamblea) o se concentran en uno (conjunto). En la mayoría de los casos, complican innecesariamente los textos; podemos titular un artículo El ensamble de arañas del páramo 
de Costa Rica o bien Las arañas del páramo de Costa Rica. Según la definición de Fauth et al. (1996) ambos significan lo mismo, pero por el principio de concisión (CSE 2006), el segundo título es preferible al primero. Según el principio de darse a entender, Relaciones tróficas de las arañas del páramo de Costa Rica sería aún mejor.

\section{AGRADECIMIENTOS}

Agradezco a Zaidett Barrientos (UNED), Vanessa Nielsen y Priscilla Carbonell (UNED) por su apoyo con la literatura y el análisis, y a Carlos Morales (UCR) por sugerencias para mejorar el manuscrito.

\section{RESUMEN}

En años recientes ha aparecido la palabra «ensamble» (con menos frecuencia, «ensamblaje») en la literatura biológica en castellano, con dos significados diferentes: grupos de especies de un hábitat relacionadas taxonómicamente; y grupos de especies de un lugar sin interacciones ecológicas. Tras analizar las definiciones de la palabra en el original francés, así como sus usos técnicos en castellano e inglés, concluyo que las palabras assemblage y ensemble tienen traducción adecuada al español, assemblage como asamblea y ensemble como conjunto, pero en la mayoría de los casos tienen un aporte dudoso y complican innecesariamente los textos, por lo que según los principio de concisión y comunicación, conviene evitarlas.
Key words: terminología ecológica, uso correcto del castellano, español biológico, definición de ensamble, ensamblaje en biología.

\section{REFERENCES}

Cooke, J. G. (1984). Glossary of technical terms. In R. M. May (Ed.), Exploitation of Marine Communities. Berlín: Springer-Verlag.

Council of Science Editors. (2006). Scientific Style And Format: The CSE Manual for Authors, Editors, And Publishers. Chicago: Council of Science Editors.

Fauth, J. E., Bernardo, J., Camara, M., Resetarits, W. J., Van Buskirk Jr., J., \& McCollum, S. A. (1996). Simplifying the Jargon of Community Ecology: A Conceptual Approach. The American Naturalist 147: 282-286

Jaksić, F.M. (1981). Misuse of the Term "Guild" in Ecological Studies. Oikos 37(3): 397-400.

McCune, B., Grace, J. B., \& Urban, D. L. (2002). Analysis of Ecological Communities. Gleneden Beach, Oregon: MjM Software Design.

Moreira, F. M. S., Huising, E. J., \& Bignell, D. E. (2012). Manual de biología de suelos tropicales. México DF: Secretaría de Medio Ambiente y Recursos Naturales (SEMARNAT) e Instituto Nacional de Ecología (INE).

Quijada, P. A. \& Cáceres, C. W. (2000). Patrones de abundancia, composición trófica y distribución espacial del ensamble de peces intermareales de la zona centro-sur de Chile. Revista Chilena de Historia Natural, 73: 739-747.

Verhoef, H. E. \& Morin, P. J. (2009). Community Ecology: Processes, Models, and Applications. Oxford: Oxford University. 
